\title{
Atrial septal defect presenting as recurrent primary amoebic lung abscess
}

\author{
Manoj Sharma, Harish Mehta and S.K. Sharma \\ Department of Cardiology, BMRC, SMS Medical College, Jaipur, India
}

\begin{abstract}
Summary: A middle-aged female with an atrial septal defect (secundum type) presented with a primary pulmonary amoebic abscess. She was successfully treated with antiamoebic therapy. One year later she presented with a similar lung abscess which again responded to antiamboebic treatment. Attention is drawn to the fact that a patient with a left to right shunt can present with a recurrent rare primary parasitic infection of the lung.
\end{abstract}

\section{Introduction}

Primary pulmonary amoebiasis is $\operatorname{rare}^{1-3}$ and usually occurs as a result of haematogenous spread from the colon without involving the liver. ${ }^{1}$ It may also occur following inhalation of dust containing cysts of Entamoeba histolytica. ${ }^{4}$ Increased susceptibility to pulmonary infections has been described in patients with atrial septal defect (ASD) with left to right shunt. ${ }^{5,6}$ We report a patient with ASD with recurrent primary amoebic pulmonary abscess.

\section{Case report}

A 52 year old female presented with high grade fever, cough with chocolate-brown expectoration and severe dyspnoea for one week. On general physical examination she had tachycardia, tachypnoea, temperature $38.2^{\circ} \mathrm{C}$, ankle oedema and raised jugular venous pressure. Respiratory system examination revealed signs suggestive of a cavitatory lesion in the lower lobe of the right lung. On cardiovascular examination, the second heart sound was widely and fixed split and an ejection systolic murmur of grade III/VI was best heard in the pulmonary area. Tender hepatomegaly was present.

Investigation showed neutrophilic leucocytosis with a very high erythrocyte sedimentation rate. The liver function tests were within the normal range. Chest X-ray showed a cavitatory lesion with air fluid level in the posterobasal region of the lower lobe of the right lung (Figure 1). The diaphragm was freely mobile on fluoroscopy. No focal lesion

Correspondence: S.K. Sharma, M.D., F.I.C.A., E-2, Shanti Path, Tilak Nagar, Jaipur 302004, India

Accepted: 10 November 1990

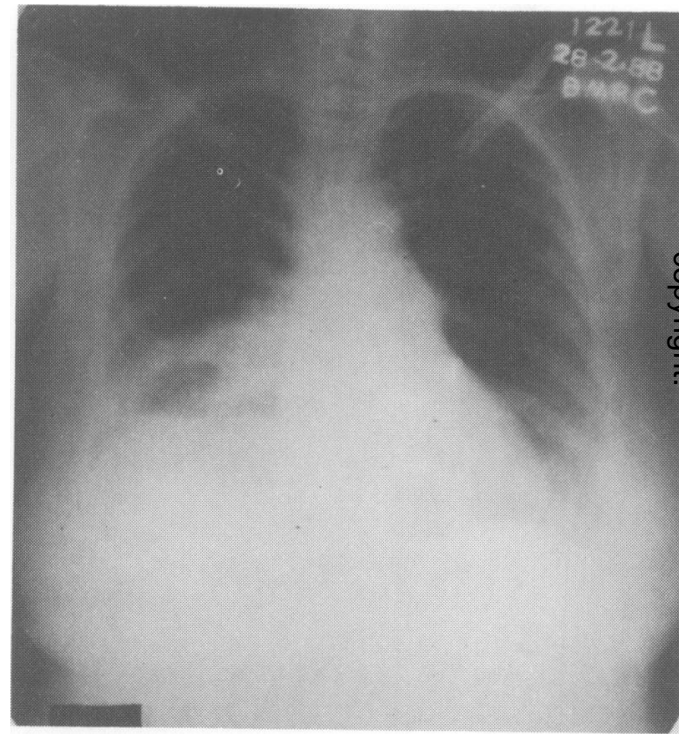

Figure 1 X-ray chest (PA view) showing a homogenous opacity with air fluid in right lower lobe.

was seen on ultrasonography or technetium-99m scan of the liver. Bronchoscopic aspirate and washings showed pus cells only, and no bacteria acid-fast bacilli, Entamoeba histolytica, liver cells or malignant cells were seen. No organisms were grown on culture. Indirect haemagglutination (IHA) test for amoebiasis was positive in 1:2048 dilution. 2-D echocardiogram confirmed an atrial septal defect of secundum type and right ventricular overload.

The patient showed remarkable clinical and radiological improvement on treatment with metronidazole and dehydroemetine followed by chloroquine for 3 weeks. Almost a year later, she presented again with similar complaints, and 
clinical and radiological evidence of right lung abscess. Amoebic serology (IHA) was positive in 1:2048 dilution and she responded to metronidazole, dehydroemetine and chloroquine.

\section{Discussion}

Amoebic lung abscess usually occurs as a result of direct extension of liver abscess. ${ }^{2}$ The diaphragm in these cases is adherent to the under surface of lungs and pleura. ${ }^{1}$ Primary pulmonary amoebiasis is rare $^{1,3}$ and occurs as a result of haematogenous spread from the colon without involving the liver. ${ }^{1}$ The infection is likely to establish itself following entry of trophozoites into branches of the middle and inferior haemorrhoidal or vertebral system of veins into the inferior vena cava and then reaching the pulmonary circulation. ${ }^{2}$ It may also occur following inhalation of dust containing cysts of $E$. histolytica. ${ }^{4}$ The sputum does not necessarily show amoebae $^{3}$ and the diagnosis in these cases rests on the combination of suggestive clinical and

\section{References}

1. Webster, B.H. Pulmonary complications of amoebiasis. Dis Chest 1956, 30: 315-325.

2. Vyas, P.N., Bhargava, N.N. \& Gopinathan, N. Pleuropulmonary amoebiasis. Ind J Surg 1963, 25: 141-149.

3. Manson-Bahr, P.H. Pulmonary amoebiasis. In: Manson's Tropical Diseases, 16th ed. Bailliere, Tindall \& Cassell, London, 1966, pp. 458-459.

4. Sayyed, E.W. Pulmonary amoebiasis of the upper lobe. Dis Chest 1963, 44: 325-328. laboratory findings with a definite and dramatic response to antiamoebic therapy. ${ }^{2,3}$

In the present case the amoebic nature of the lung abscess was established by the nature of the sputum, serology and therapeutic response. However, it did not seem to be a secondary event, because there was no detectable hepatic involvement, either sonographically or radioisotopically, nor was the diaphragm adherent to the undersurface of the lung and pleura. The hepatic enlargement in this patient was attributed to congestive heart failure secondary to the ASD. Concurrent anti-congestive therapy was also administered resulting in normalization of the central venous pressure and regression of hepatomegaly. It therefore seems that the pulmonary abscess was a primary amoebic infection of the lung. Patients with ASD with a left to right shunt are known to suffer from an increased incidence of pulmonary infections. ${ }^{5,6}$ However, to the best of our knowledge we know of no case of ASD, presenting with a pulmonary infection due to $E$. histolytica.

5. Haroutonian, L.M. \& Catherine, A.N. Pulmonary complications of congenital heart disease. Am Heart $J$ 1972, 84: 540-559.

6. Braunwald, E. \& Borow, K.M. Congenital heart disease in the adult. In: Braunwald, E. (ed.). Braunwald Heart Disease, a Text Book of Cardiovascular Medicine, third edition. W.B. Saunders, Philadelphia, 1988, p. 976. 